\title{
Xyloglucan endotransglucosylase and cell wall extensibility
}

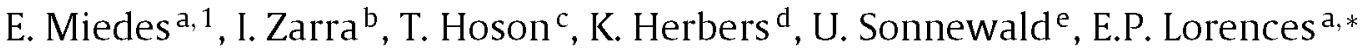 \\ a Dpto Biologia Vegetal. Facultad de Farmacia. Universidad de Valencia. 46100 Burjassot Valencia. Spain \\ ${ }^{\mathrm{b}}$ Dpto. Fisiología Vegetal. Facultad de Biológicas. Universidad de Santiago de Compostela. 15782 Santiago de Compostela. Spain \\ ${ }^{-}$Dept. Biological Sciences. Graduate School of Science. Osaka City University. Sumiyoshi-ku, 558-8585 Osaka. Japan \\ ${ }^{d}$ BASF Plant Science. BPS-L444. 67117 Limburgerhof. Germany \\ ${ }^{\mathrm{e}}$ Lehrstuhl für Biochemie. Institut für Biologie. Universität Erlangen-Nümberg. Staudtstr. 5. 91058 Erlangen. Germany
}

Keywords:

Cell wall. Extensibility. Growth. Solanum lycopersicum L. cv. Money Maker.

Xyloglucan

\section{S U M M A R Y}

Transgenic tomato hypocotyls with altered levels of an XTH gene were used to study how XET activity could affect the hypocotyl growth and cell wall extensibility. Transgenic hypocotyls showed significant over-expression (line 13) or co-suppression (line 33) of the SIXTH1 in comparison with the wild type, with these results being correlated with the results on specific soluble XET activity, suggesting that SIXTH1 translates mainly for a soluble XET isoenzyme. A relationship between XET activity and cell wall extensibility was found, and the highest total extensibility was located in the apical hypocotyl segment of the over-expressing SIXTH1 line, where the XET-specific activity and hypocotyl growth were also highest compared with the wild line. Also, in the co-suppression SIXTH1 line, total extensibility values were lower than in the wild type line. The study of linkages between cell wall polysaccharides by FTIR showed that hypocotyls over-expressing SIXTH1 and having a higher XET-specific activity, were grouped away from the wild line, indicating that the linkages between pectins and between cellulose and xyloglucans might differ. These results suggested that the action of the increased XET activity in the transgenic line could be responsible for the cell wall structural changes, and therefore, alter the cell wall extensibility. On the other hand, results on xyloglucan oligosaccharides composition of the xyloglucan by MALDI TOF-MS showed no differences between lines, indicating that the xyloglucan structure was not affected by the XET action. These results provide evidences that XTHs from group I are involved mainly in the restructuring of the cell wall during growth and development, but they are not the limiting factor for plant growth.

\section{Introduction}

The plant cell wall plays a vital role in controlling the shape of individual cells and, therefore, in determining the morphology of the plant as a whole. Modifications to plant primary cell walls are required for many processes, such as plant cell expansion and growth, as well as other developmental processes that require cell wall degradation, such as fruit ripening and organ abscission (Rose and Bennet, 1999). The primary cell walls of flowering plants consist of a framework of cellulose embedded in a matrix of hemicelluloses, pectins and structural proteins (Carpita and Gibeaut,
1993). The most abundant hemicellulose of the dicotyledonous wall is xyloglucan; this long polysaccharide can form hydrogen bonds with cellulose microfibrils and might provide a molecular tether between adjacent microfibrils (Hayashi, 1989).

Cell elongation and expansion in plants is a complex but wellcoordinated process. During elongation, a biochemical change in wall extensibility allows turgor pressure to drive cell expansion, and the deposition of new wall materials maintains microfibril spacing and wall thickness and irreversibly extends the walls, making the wall compartment larger (Taiz, 1984; Cosgrove, 1999). Controlled loosening of the cellulose-xyloglucan network may occur by way of mechanisms that modify the xyloglucan cross-links directly or indirectly. The basis of xyloglucan metabolism during cell elongation and the biochemical basis of changes in wall extensibility are still not clear and remain largely uncharacterized. The involvement of several classes of enzymes has been suggested, including endo-1,4- $\beta$-D-glucanases (EGases), xyloglucan endotransglycosylases/hydrolases (XTHs) and exo-glycosylhydrolases (glycosidases) (Fry, 2004).

Xyloglucan endotransglycosylase/hydrolase (XTH) has been considered as an important candidate as a wall-loosening enzyme (Fry et al., 1992; Nishitani and Tominaga, 1992). XTH can act as 
a transglycosylase (XET), having a dual role, integrating newly secreted xyloglucan chains into an existing wall-bound xyloglucan, restructuring existing cell wall material by catalyzing transglycosylation between previously wall bound xyloglucan molecules, or acting as hydrolase (XEH), hydrolyzing one xyloglucan molecule, depending on the nature of the xyloglucan donor and acceptor substrates (Fry et al., 1992; Nishitani and Tominaga, 1992; Rose et al., 2002).

It has been reported that spatial distribution of XTH expression and XET activities are closely correlated with both cell elongation (Fry, 1992; Catalá et al., 1997; Vissenberg et al., 2000; Ji et al., 2003) and the non-elongation zone (Vissenberg et al., 2001; Takeda et al., 2002), even during secondary cell wall formation (Antosiewicz et al., 1997; Bourquin et al., 2002; Matsui et al., 2005).

Furthermore, XET may be important for regulating the polymer length and insertion of xyloglucans into the cell wall, which could alter the extensibility of the cell wall (Fry et al., 1992; Nishitani and Tominaga, 1992; Thompson and Fry, 2001). Although XET failed to cause cucumber cell walls to extend in an in vitro assay (McQueenMason et al., 1993), other reports have suggested that XET could be involved in cell wall extensibility. A decrease in XET activity was reported in primary roots of maize with low water potential, which was correlated with a decrease in cell wall extensibility and cell elongation in that region (Pritchard et al., 1993). Also, Wu et al. $(1994,1996)$ found enhanced XET activity that was correlated with an increase in cell wall extensibility. These results strongly support a potential role of XET associated with cell wall extensibility and, thus, cell elongation.

The different XTHs have been grouped into four groups for Arabidopsis thaliana (Yokoyama and Nishitani, 2001) and into three groups for Solanum lycopersicum (Saladie et al., 2006). From the 25 SIXTHs genes reported, SIXTH1 has been associated with hypocotyl growth, and it seems to show only XET activity (Catalá et al., 1997, 2001)

The objective of this work was to further study the effect of the $X T H$ in cell wall extensibility. The work included the generation of transgenic tomatoes with altered levels of an XTH gene related to tomato hypocotyl growth (SIXTH1), to increase or to decrease the XET activity in tomato hypocotyls, and evaluate how XET activity could affect hypocotyl growth and cell wall extensibility.

\section{Materials and methods}

\subsection{Tomato transformation}

The plants were transformed using Agrobacterium-mediated gene transfer using strain C58C1:pGV2260 (Rosahl et al., 1987). Transgenic tomato plants expressing an XTH (acc. no.: D86730) from Nicotiana tabacum under the control of the CaMV-35S promoter were created as described in Herbers et al. (2001). Transformation of plants was performed essentially as described by Ling et al. (1998) using tomato cotyledons and a tobacco cell suspension culture as a feeder layer. Screening was perfomed for approximately 50 transgenic lines.

\subsection{Plant material and growth conditions}

Homozygotic tomato seeds (Solanum lycopersicum L. cv. Money Maker) were grown in the dark at $25^{\circ} \mathrm{C}$ and $70 \%$ relative humidity. One hundred fifty seeds were sown in staggered formation into a tray $(43 \times 28 \times 7 \mathrm{~cm})$ with 4 filter papers moistened with $80 \mathrm{ml}$ milliQ water. In most experiments, etiolated hypocotyls were grown for 6 days and were selected when $25-35 \mathrm{~mm}$ in length. Two $10 \mathrm{~mm}$ segments were cut from the hypocotyls: the apical segment below the hook, and the basal segment below the apical.

\subsection{RNA extraction and Northern blot analysis}

RNA extraction and Northern analysis were performed as described by Herbers et al. (1994). Northern blots were hybridized with the complete cDNA from NtXET1 or SIXTH1 (D16456).

\subsection{Real-Time quantitative $P C R$}

Apical and basal hypocotyls segments (approximately $100 \mathrm{mg}$ ) were harvested and mRNA extraction, cDNA preparation and Real-Time quantitative PCR were performed as described by Miedes and Lorences (2009). The PCR amplification was performed with a specific primer for the SIXTH1 gene. Because the homology between NtXET1 and SIXTH1 is very high (88\%), the primers for real time RT-PCR were designed for a region that showed differences in sequence (Fig. 1). The primer sequence (forward/reverse) was CTTGAGAAAACCAATTGGGCCAAC/GAACCCAACGAAGTCTCCTATACTGTAATG, and for RPL2 (a housekeeping gene, ribosomal protein large subunit 2), used as internal control for the gene, ATTCACGATCCAGGGAGAGGTGC/AGGCAACACGTTACCAACCATAAGAGTAG. Three replicates were performed for each sample in $25 \mu \mathrm{l}$ final volume containing $1 \mu \mathrm{l}$ CDNA, 25 pmol specific primers, and $12.5 \mu \mathrm{l}$ of PowerSYBR Green PCR Master Mix according to the manufacturer's instructions.

\subsection{Protein extraction and xyloglucan endotransglycosylase (XET) assay}

Soluble and ionically cell wall bound proteins were extracted together by homogenization of $35 \mathrm{mg}$ apical and basal hypocotyl segments, with $40 \mu \mathrm{L}$ of sodium phosphate $200 \mathrm{mM}(\mathrm{pH} 6.0)$ at $4^{\circ} \mathrm{C}$, and then centrifuged at $10,000 \mathrm{~g}$ for $10 \mathrm{~min}$ at $4^{\circ} \mathrm{C}$. After centrifugation, the supernatant (crude enzyme extract) was used for the assay of soluble XET activity. The protein content of crude extract was determined using the Coomassie Blue $G$ dye-binding method (Bradford, 1976). Active cell walls were obtained from the residue and we also assayed the insoluble (cell wall-bound) XET. Soluble XET was assayed as described previously (Fry et al., 1992), and $\left[{ }^{3} \mathrm{H}\right] \mathrm{XXXGol}$ was used as the acceptor for the endotransglycosylation reaction. Xyloglucan heptasaccharide XXXG was prepared as described previously (Lorences and Fry, 1993). $\left[{ }^{3} \mathrm{H}\right] \mathrm{XXXGol}$ was prepared by reduction of the reducing terminal glucose moiety of non-radioactive XXXG with $\left[{ }^{3} \mathrm{H}\right] \mathrm{NaB}_{3} \mathrm{H}_{4}$. The solution of $\left[{ }^{3} \mathrm{H}\right] X X X \mathrm{Gol}$ used for the XET assays had a specific activity of 22.5 $\mathrm{TBq} \mathrm{mol}^{-1}$. Soluble reaction mixtures (total volume $40 \mu \mathrm{l}$ ) containing $5 \mathrm{mg} \mathrm{ml}^{-1}$ of partially purified apple xyloglucan, $0.85 \mathrm{kBq}$ [3H]XXXGol, $50 \mathrm{mM}-\mathrm{MES}\left(\mathrm{Na}^{+}\right) \mathrm{pH} 6.0$ and $25 \mu \mathrm{l}$ of enzymatic extract $\left(0.5-0.6 \mathrm{mg} \mathrm{ml}^{-1}\right)$ were incubated for one hour at $25^{\circ} \mathrm{C}$. The reaction was stopped by the addition of $100 \mu \mathrm{l}$ of $20 \%(\mathrm{w} / \mathrm{v})$ formic acid and the solution was then dried on $5 \mathrm{~cm} \times 5 \mathrm{~cm}$ Whatman 3MM filter paper, washed for $30 \mathrm{~min}$ in running tap water to remove unchanged $\left[{ }^{3} \mathrm{H}\right] \mathrm{XXXGol}$, re-dried and assayed for ${ }^{3} \mathrm{H}$ by scintillation counting. Insoluble XET was assayed as described by Barrachina and Lorences (1998). Reaction mixtures containing $2 \mathrm{mg}$ of enzymatic active cell wall, $40 \mu 150 \mathrm{mM}$-MES $\left(\mathrm{Na}^{+}\right) \mathrm{pH} 6.0$ and $0.85 \mathrm{kBq}[3 \mathrm{H}] \mathrm{XXXGol}$ were incubated for one hour at $25^{\circ} \mathrm{C}$. The reaction was stopped by the addition of $100 \mu \mathrm{l}$ of $20 \%$ (w/v) formic acid and was centrifuged. The residue was washed with distilled water and then the cell wall was hydrolyzed with $200 \mu$ l trifluoracetic acid $2 \mathrm{~N}$ for 1 hour at $121^{\circ} \mathrm{C}$. The residue was then assayed for ${ }^{3} \mathrm{H}$ by liquid scintillation counting. Inactivated controls were carried out in the same way using enzyme previously boiled for $30 \mathrm{~min}$. 


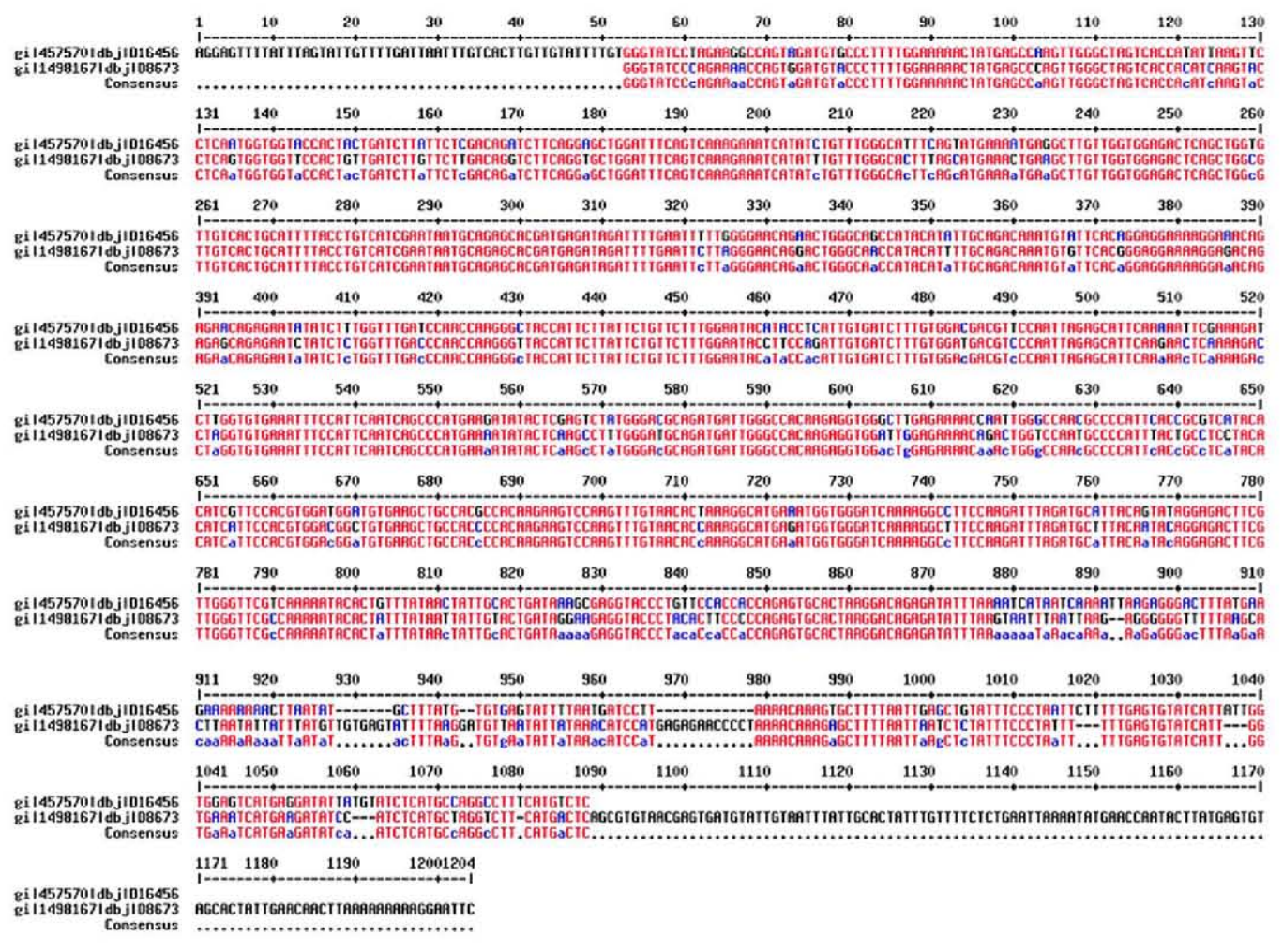

Figure 1. Alignment of SIXTH1 and NtXET1 sequences.

\subsection{Plant growth}

Etiolated hypocotyl length was measured daily after germination until 14 days of growth, using a flexible ruler. Manipulations were performed under dim green light $\left(0.02 \mathrm{~W} \mathrm{~m}^{2}\right)$. Kodak safelight Filter $\mathrm{N}^{\circ}$ 3, NY, EEUU).

\subsection{Cell wall extensibility}

Apical and basal hypocotyl segments were boiled in methanol for $10 \mathrm{~min}$ and stored in fresh methanol until use. Before the measurement of the cell wall extensibility, the fix cell wall was rehydrated for 1 hour at $4{ }^{\circ} \mathrm{C}$ with several changes of distilled water. The cell wall extensibility was measured with a tensile tester (TensilonRTM-25, Toyo Baldwin Co.Tokio, Japan). The sample was fixed between two clamps (the distance between the clamps was $5 \mathrm{~mm}$ ) and stretched by lowering the bottom clamp at a speed of $10 \mathrm{~mm} \mathrm{~min}^{-1}$ until a stress of $8 \mathrm{~g}$ was produced. The total extensibility was measured with the first strain/stress curve immediately before the stress of $8 \mathrm{~g}$ was produced, and the reversible extensibility with that of the second curve. The irreversible extensibility was calculated as the difference between the total extensibility and reversible extensibility (Soga et al., 2001).

\subsection{Fourier Transform Infrared Spectroscopy (FTIR)}

Apical segments from 6-day-old hypocotyls were selected for FTIR. Samples of 10 segments were dehydrated by shake washing with methanol:chloroform $(1: 1 ; v: v)$ and finally with diethyl ether. The samples were then dried at room temperature and kept in a vacuum oven at $70^{\circ} \mathrm{C}$. Tablets for FTIR spectroscopy were prepared in a manual press using cell walls (2 mg) mixed with $\operatorname{KBr}(1: 100$ $\mathrm{p} / \mathrm{p})$. Spectra were obtained on a Bomen-Michelson instrument, where one hundred twenty-eight interferograms were collected in transmission mode, with $4 \mathrm{~cm}^{-1}$ resolution, for each sample. A window between 750 and $2200 \mathrm{~cm}^{-1}$, which contains information on characteristic polysaccharides, was selected to monitor cell wall structure modifications. All spectra were normalized and baselinecorrected as described elsewhere (Robin et al., 2003). Data were then exported to Win-Das (Kemsley, 1998) and all spectra were area-normalized $\left(800-1800 \mathrm{~cm}^{-1}\right)$. Principal component analysis (PCA) was performed using a maximum of five principal components.

\subsection{Matrix Assisted Laser Desorption Ionization Time of flight Mass Spectrometry (MALDI TOF-MS) analysis of xyloglucan oligosaccharides}

Apical segments from 6-day-old hypocotyls (c.a. $100 \mathrm{~g}$ ) were selected for mass spectrometry analysis. The tissue was homogenize, using an IKA Ultraturrax T25 (Stanfen, Germany) in 100\% methanol and then boiled for $30 \mathrm{~min}$. Then homogenate was washed twice with methanol, acetone, methanol:chloroform $(1: 1$; $\mathrm{v}: \mathrm{v})$ and finally with diethyl ether. The cell wall material pellet was dried under a vacuum. Cell wall residue $(2 \mathrm{mg})$ was then treated with 2.5 units of a cellulase Trichoderma sp. (EC 3.2.1.4; Megazyme. Bray, Ireland) in $1 \mathrm{ml}$ of $10 \mathrm{mM}$ acetic-pyridine $\mathrm{pH} 4.7$, at $37^{\circ} \mathrm{C}$ in an orbital shaker at $150 \mathrm{~g}$ for 18 hours. The undigested wall material was removed by centrifugation at $10,000 \mathrm{~g}$ for $5 \mathrm{~min}$. The supernatant $(100 \mu \mathrm{l})$ was transferred and filtrated to a fresh tube (Ultrafree MC 0.45 Millipore with Q-sepharose Fast Flor Pharmacia Biotech). Then, the samples were vacuum-dried (RC 10.10 Jovan, England) and solubilized in 101 milliQ water. An aliquot $(2 \mu \mathrm{l})$ of the solution was mixed with the same volume of $10 \mathrm{mg} \mathrm{ml}^{-1} 2,5-$ dihydroxycinamic acid in acetonitrile:water $(7: 3, v / v)$ and then, $1 \mu \mathrm{L}$ of mix was spotted onto dried on a target plate. The sample was analyzed by MALDI-TOF mass spectrometry (Autoreflex MALDI TOF-MS Broker Daltonics, Bremen, Germany) operated in the positive-ion mode with an accelerating voltage of $19 \mathrm{kV}$ and a delay time of $150 \mathrm{~ns}$ in reflector mode. Automatic modus accumulated 8 spectra per sample, with each spectrum originating from between 200-300 laser pulses. The MS spectra obtained were com- 


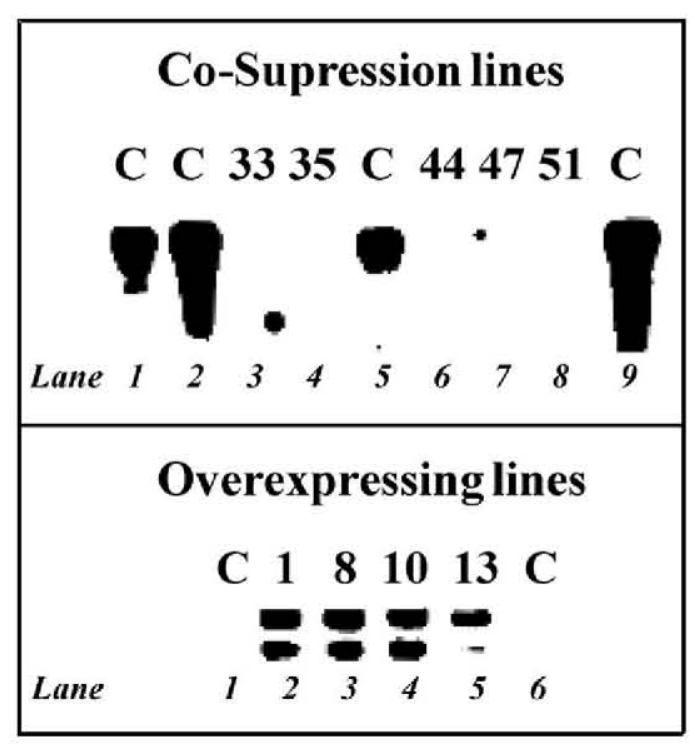

Figure 2. Northern analysis of XTH mRNA in transgenic tomato plants. Specific probes for CDNA from NtXET1 were used. The five independent co-suppression lines: 33 (lane 3), 35 (lane 4), 44 (lane 6), 47 (lane 7) and 51 (lane 8) are compared with four wild type plants (lames 1, 2, 5, 9). The four over-expressing lines: 1 (lane 2), 8 (lane 3), 10 (lane 4), and 13 (lane 5) are compared with two wild type plants (lanes 1,6).

pared to highlight significantly increased or decreased relative peak areas.

\section{Results}

\subsection{Tomato plants transformation. Expression analysis of SLXTH1 and NTXET1}

After the transformation of the tomato plants, a screening of approximately 50 transgenic lines using cDNA probes from NtXET1 or SIXTH1 was performed (Fig. 1). Lines 1, 8, 10, and 13 showed a reasonable over-expression of NtXET1 RNA as demonstrated by northern blotting. Lines $1,8,10$, and to a lesser extent, the 13th line, showed two bands, the upper band being the one corresponding to NtXET1 plus ocs terminator (930 bp); the smaller mRNA might result from premature transcriptional termination. Line 13 was selected for further experiments. Lines $33,35,44,47$ and 51 showed a co-suppression of NtXET1 RNA (Fig. 2), and line 33 was selected for further experiments

The results of the expression analysis of the SIXTH1 gene by Real-Time quantitative PCR of the wild type and the selected overexpressing and co-suppression lines are shown in Figure 3. The results showed that, in the apical hypocotyl segments, the expression of SIXTH1 was higher than in the basal segments in all the studied lines, the wild type and the transgenic lines. In the apical segments of the over-expressing line (line 13), expression of the SIXTH1 gene was higher compared to the wild type. Although primers were specific for SIXTH1 amplification, some contribution of the over-expressed NtXET1 cannot be excluded as a possibility. On the other hand, in the co-suppression line (line 33), the expression of SIXTH1 was much lower (26\%) than in the wild type. In the basal hypocotyl segments, the relative expression of SIXTH1 was lower than in the apical segment, as noted above, and we again found a significant increase in SIXTH1 expression in the overexpressing line and a decrease in the co-suppression line compared to wild type.

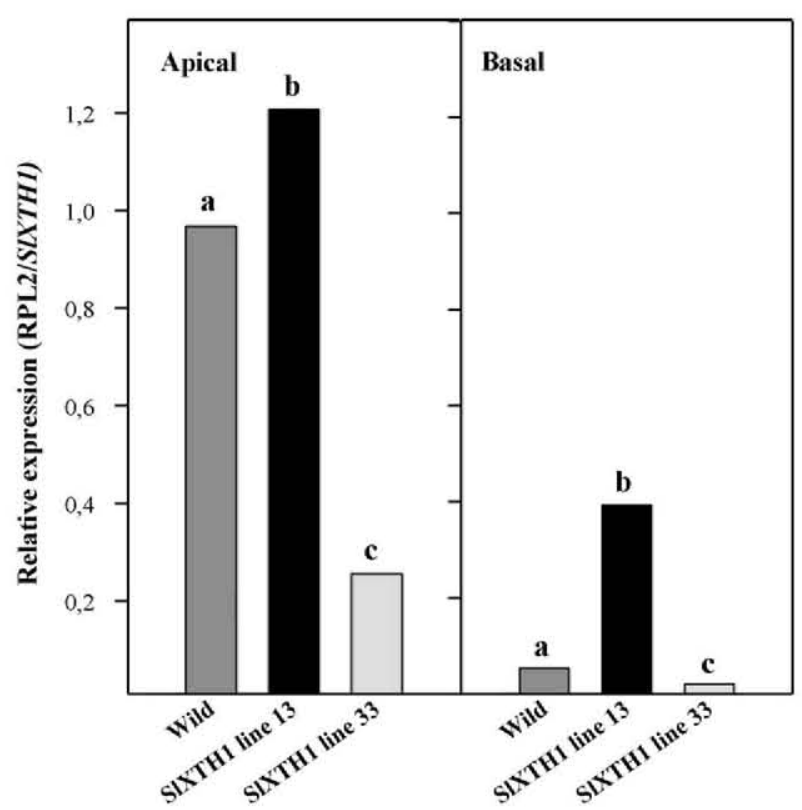

Figure 3. Expression analysis of SIXTH1 tomato gene by Real-Time quantitative PCR from apical and basal hypocotyls segments of wild type and transgenic tomato lines. Values are means of 2 independent experiments using 20 hypocotyls from each determination $\pm S E$. Different letters indicate significant differences between wild type and transgenic tomato lines (T-test, p-value $<0.05$ ).

\subsection{XET activity during elongation}

Soluble and insoluble endotransglucosylase activities were determined in the apical and basal hypocotyl segments of tomato using $\left[{ }^{3} \mathrm{H}\right] \mathrm{XXXGol}$ as the acceptor for the endotransglucosylation reaction (Figure 4). The results showed that both XET activities in apical hypocotyl segments were higher than the basal hypocotyl segments in all lines. The soluble XET activity in the apical hypocotyl segment was significantly higher in the over-

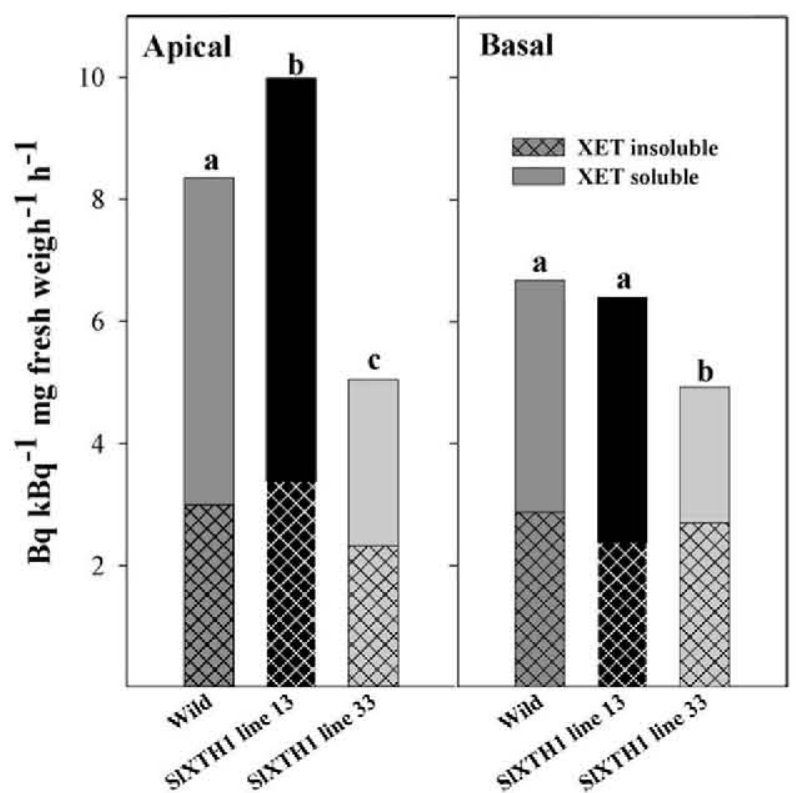

Figure 4. Soluble and insoluble activities of xyloglucan endotransglucosylase (XET) from apical and basal hypocotyls segments of wild type and transgenic tomato lines. The XET assay was performed by measuring $\left[{ }^{3} \mathrm{H}\right] \mathrm{XXXG}$ incorporation into xyloglucan by the protein extracts. Values are means of 5 independent experiments using 20 hypocotyls from each determination. Different letters indicate significant differences between wild type and transgenic tomato lines ( $t$-test, $p$-value $<0.05$ ). 


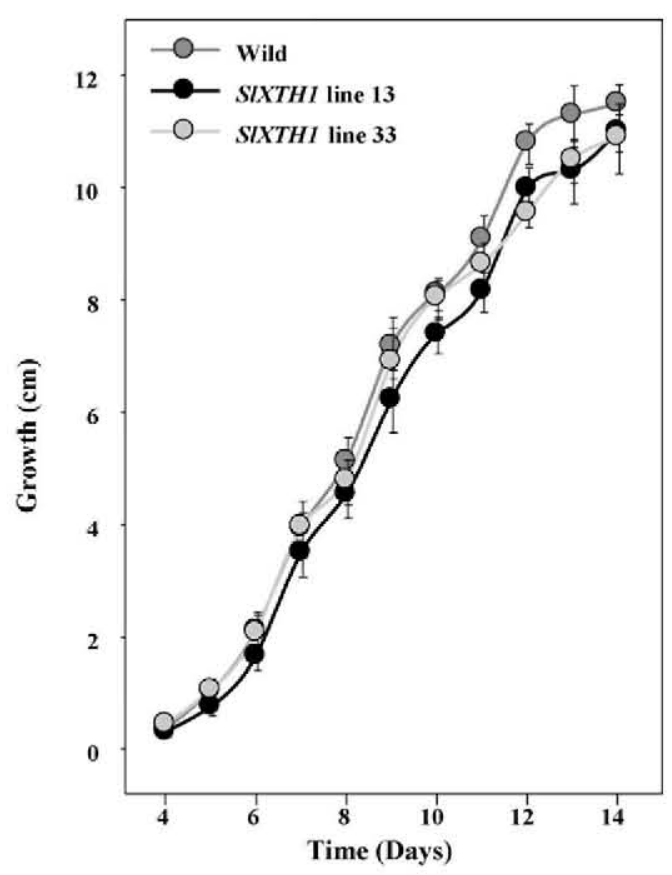

Figure 5. Kinetic of etiolated hypocotyls growth from wild type and transgenic tomato lines. The length of hypocotyls was measured after germination. Values are means of 5 independent experiments using 40 hypocotyls from each determination \pm SE.

expression line than in the wild type, while soluble XET activity in the co-suppression line was significantly lower than in the wild type line. Soluble XET activity in basal hypocotyl segments was similar in wild types and in line13, and significantly lower in line 33. With respect to insoluble XET activity, in the apical hypocotyls segments, the differences in activity among the three lines followed the same pattern as the soluble XET activity, although the results did not show significant different among the three lines, and no differences were found in the insoluble XET activity in the basal hypocotyls segments.

\subsection{Hypocotyl growth.}

Hypocotyl growth after germination is shown in Figure 5, and the results showed no differences between the wild and the transgenic tomato lines.

\subsection{Determination of the mechanical properties of cell wall.}

In Figure 6, we show the total, reversible and irreversible extensibility of apical and basal parts of etiolated hypocotyls. Apical extensibility results were higher than basal extensibility in all lines. In the apical part, total extensibility of SIXTH1 line 13 was higher than SIXTH1 line 33 and, the wild type did not differ significantly from transgenic lines. This difference in total extensibility led to the highest reversible extensibility of over-expression line 13 . In the basal part, we did not observe significant differences in mechanical properties of hypocotyls.

\subsection{Analysis of cell wall components links by FTIR}

We analyzed 15 and 19 representative FTIR cell wall spectra from the apical hypocotyl segments of wild type and transgenic lines (Figure 7), respectively. The FTIR spectra showed significant variability for the fingerprint $1600-1700 \mathrm{~cm}^{-1}$ and $800-1200 \mathrm{~cm}^{-1}$ regions. The first corresponded to esters. The second area was more difficult to associate with the differences with cell wall compo-

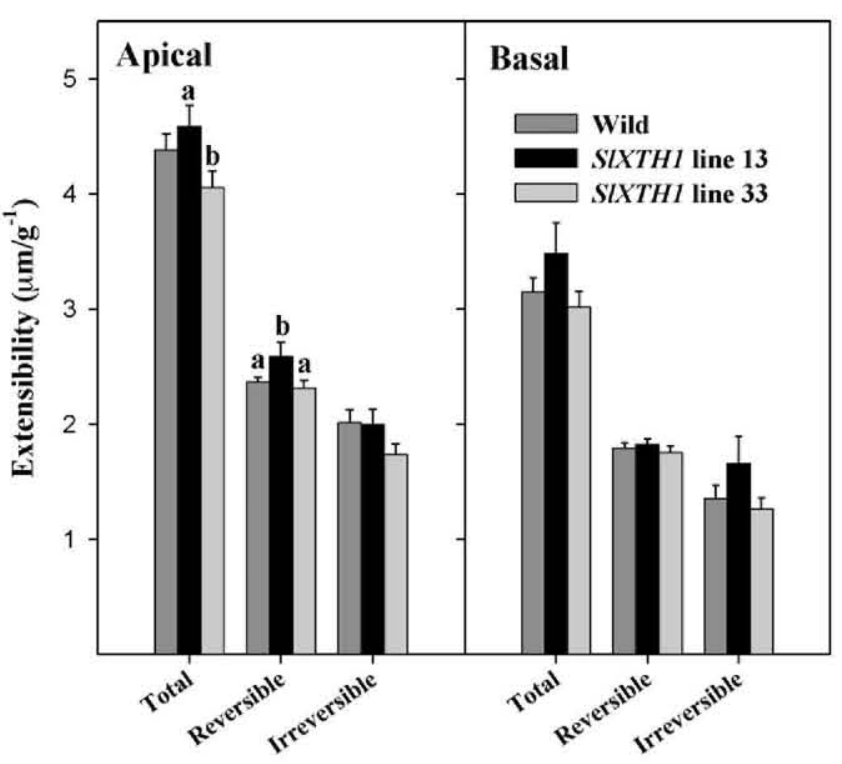

Figure 6. Extensibility of cell walls from apical and basal hypocotyls segments of wild type and transgenic tomato lines. Total, reversible and irreversible extensibility was determined. Values are means of 5 independent experiments using 30 hypocotyls from 5 different harvests in each determination \pm SE. Different letters indicate significant differences between wild type and transgenic tomato lines ( $t$ test, $\mathrm{p}$-value $<0.05$ ).

nents, due to high number of vibration modes in polysaccharide links (Sene et al., 1994). To elucidate differences among these spectra, a set of principal components analysis (PCA) was performed. The resultant spectra of sample were normalized and analyzed by the WinDas program. Principal components 1 and 2 (PC1 and PC2, which explain the $67.63 \%$ and $14.77 \%$ of total covariance of dates) could be used to separate the two groups of spectra (Figure 8 ). There was a gradient from the negative side of both principal components, occupied by over-expression of the SIXTH1 line and to the positive side of both PCs, which contained the spectra from wild type and co-suppression SIXTH1 lines. Nevertheless, The Distances Squared Mahalanobis analysis using the first five PC resolved differences between all lines studied.

\subsection{Analysis of the oligosaccharides of xyloglucan (XGOs)}

We examined the qualitative composition of xyloglucan. We investigated, using MALDI TOF-MS, the xyloglucan oligosaccha-

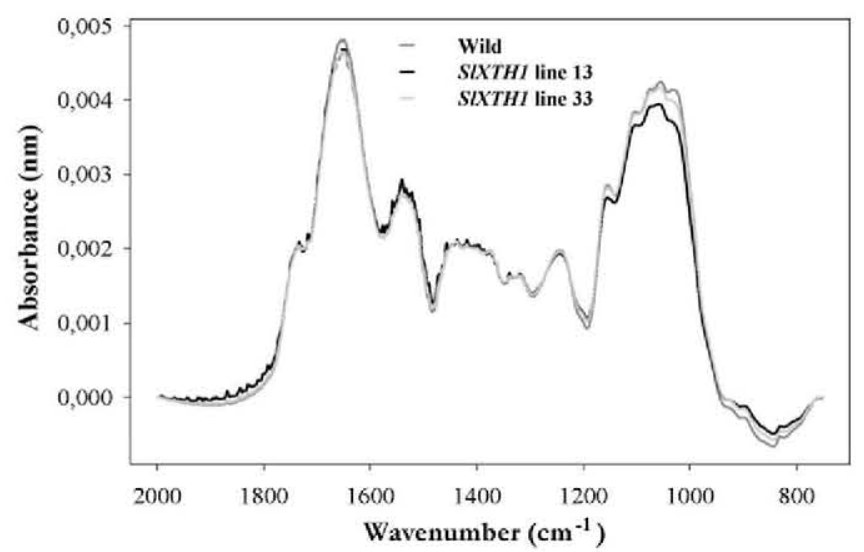

Figure 7. Cell wall infrared spectra from apical hypocotyls segments of wild and transgenic tomato lines. Values are means of 15-19 independent experiments using 10 hypocotyls from each determination. All spectra were normalized and baselinecorrected. 


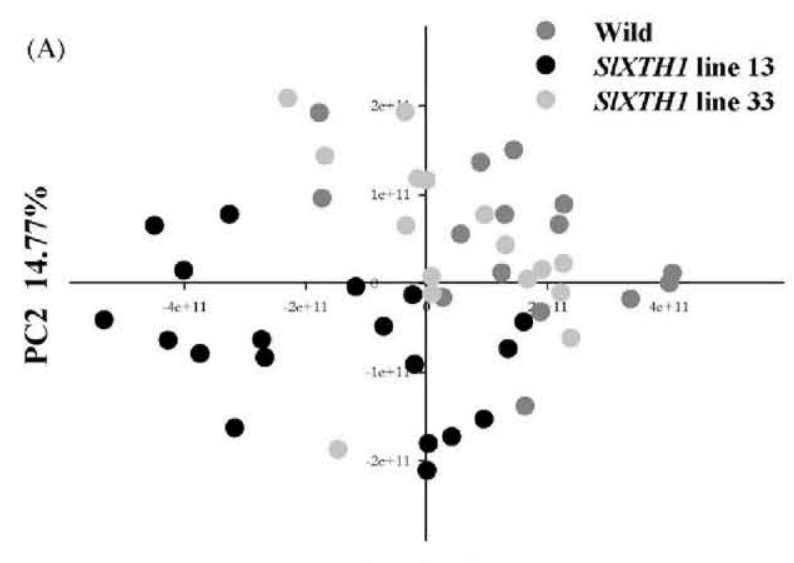

PC1 67.63\%

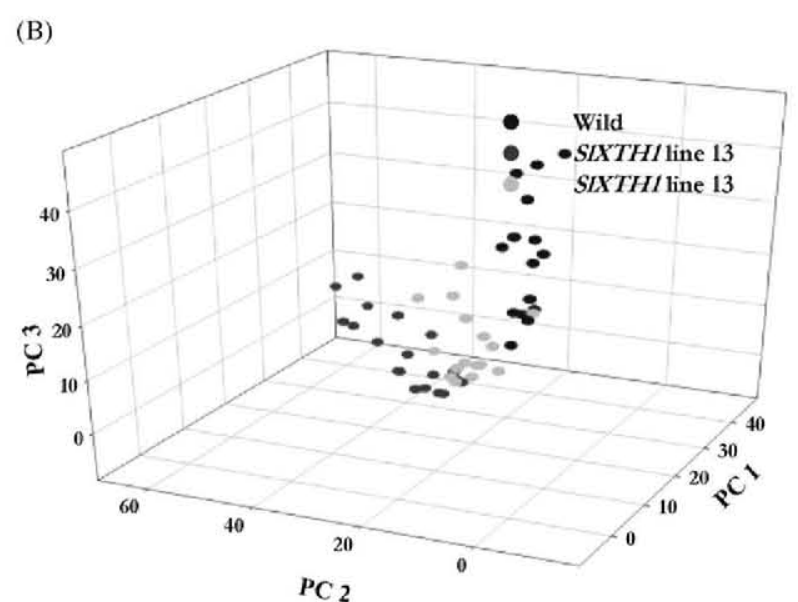

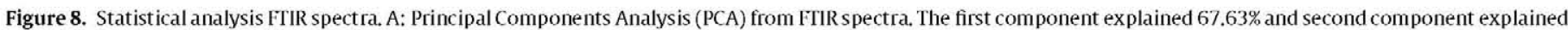

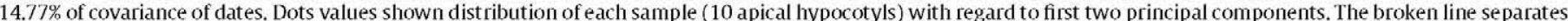
two main different populations. B: Distances Squared Mahalanobis analysis using first five principal components. The three principal components are represented.

rides released from etiolated hypocotyls tomato by endo-?-Dglucanase. First, we identified $\mathrm{m} / \mathrm{z}$ values for oligosaccharides of xyloglucan in Solanum lycopersicum L. (Table 1), since each species has their own oligosaccharides, which usually give various degrees of $\mathrm{O}$-acetylation and cations as $\mathrm{Na}^{+}$or $\mathrm{K}^{+}$associated.

\section{Table 1}

Identification of oligosaccharides of tomato xyloglucan by means of $m / z$ values, which were obtained by MALDI TOF-MS application. Most of them have complexes about acetyl groups or cations.

\begin{tabular}{|c|c|c|c|c|c|}
\hline $\mathrm{m} / \mathrm{z}$ & XGO's & $\mathrm{Ac}$ & $\mathrm{Na}+$ & $\mathrm{K}+$ & $\sum$ \\
\hline 768 & XXG (768) & & & & 768 \\
\hline 790 & XXG (768) & & 1 & & 790 \\
\hline 810 & XXG (768) & 1 & & & 810 \\
\hline 832 & XXG (768) & 1 & 1 & & 832 \\
\hline 848 & XXG (768) & 1 & 1 & 1 & 848 \\
\hline 852 & XXG (768) & 2 & & & 852 \\
\hline 874 & XXG (768) & 2 & 1 & & 874 \\
\hline 930 & XXGG (930) & & & & 930 \\
\hline 952 & XXGG (930) & & 1 & & 952 \\
\hline 995 & XXGG (930) & 1 & 1 & & 994 \\
\hline 1010 & XXGG (930) & 1 & 1 & 1 & 1010 \\
\hline \multirow{2}{*}{1018} & XXGG $(930)$ & 1 & 2 & & 1016 \\
\hline & XXGG (934) & 2 & & & 1018 \\
\hline 1033 & XXGG (930) & 1 & 2 & 1 & 1032 \\
\hline 1050 & XXGG (930) & 2 & 1 & 1 & 1052 \\
\hline 1055 & XXGG (930) & 2 & 2 & & 1058 \\
\hline 1072 & XXGG (930) & 2 & 2 & 1 & 1074 \\
\hline 1092 & LXGG (1092) & & & & 1092 \\
\hline 1062 & XSGG (1062) & & & & 1062 \\
\hline 1126 & XSGG (1062) & 1 & 1 & & 1126 \\
\hline 1142 & XSGG (1062) & 1 & 1 & 1 & 1142 \\
\hline 1156 & XSGG (1062) & 1 & 1 & 2 & 1158 \\
\hline 1211 & XSGG (1062) & 3 & 1 & & 1210 \\
\hline 1216 & XSGG (1062) & 3 & & 2 & 1220 \\
\hline 1249 & XSGG (1062) & 3 & 2 & 1 & 1248 \\
\hline 1228 & LSGG (1228) & & & & 1228 \\
\hline 1249 & LSGG (1228) & & 1 & & 1250 \\
\hline 1258 & LSGG (1228) & & & 2 & 1260 \\
\hline 1291 & LSGG (1228) & 1 & 1 & & 1292 \\
\hline 1375 & LSGG (1228) & 3 & 1 & & 1372 \\
\hline 1266 & XTGG (1198) & 1 & 1 & & 1262 \\
\hline 1288 & XTGG (1198) & 2 & & & 1282 \\
\hline 1378 & LTGG (1356) & & 1 & & 1378 \\
\hline 1420 & LTGG (1356) & 1 & 1 & & 1420 \\
\hline 1582 & XGGXXGG (1550) & & & 2 & 1582 \\
\hline 1624 & XGGXXGG (1550) & 1 & & 2 & 1624 \\
\hline 1744 & XGGXSGG (1680) & 1 & 1 & & 1744 \\
\hline \multirow[t]{2}{*}{1758} & XGGXSGG (1680) & 1 & 1 & 1 & 1760 \\
\hline & 6 Hex 5 Pen (1693) & 2 & 1 & & 1757 \\
\hline
\end{tabular}

The oligosaccharides found in tomato xyloglucan were in ascendant monosaccharide size order (one-letter abbreviation code, 40): XXG, XXGG, LXGG, XSGG, LSGG and XTGG. The relative areas of XGOǐs in apical and basal parts of hypocotyls (Figure 9) showed XSGG was the main XGO (60\% in apical and $70 \%$ in basal), followed by XXGG (40\% apical and 30\% basal). XXG and XTGG constituted only $1 \%$ of total XGOs in apical and basal segments, and LSGG was not detected in hypocotyls, but it was found in roots (data not shown). No significant differences were observed between the wild type and transgenic lines.

\section{Discussion}

As noted above, xyloglucan endotransglucosylase/hydrolase (XTH) has been considered as an important candidate as a wall loosening enzyme (Fry et al., 2002; Nishitani and Tominaga, 1992). XTH can act as a transglucosylase (XET), thus performing dual roles, integrating newly or restructuring xyloglucan molecules, or acting

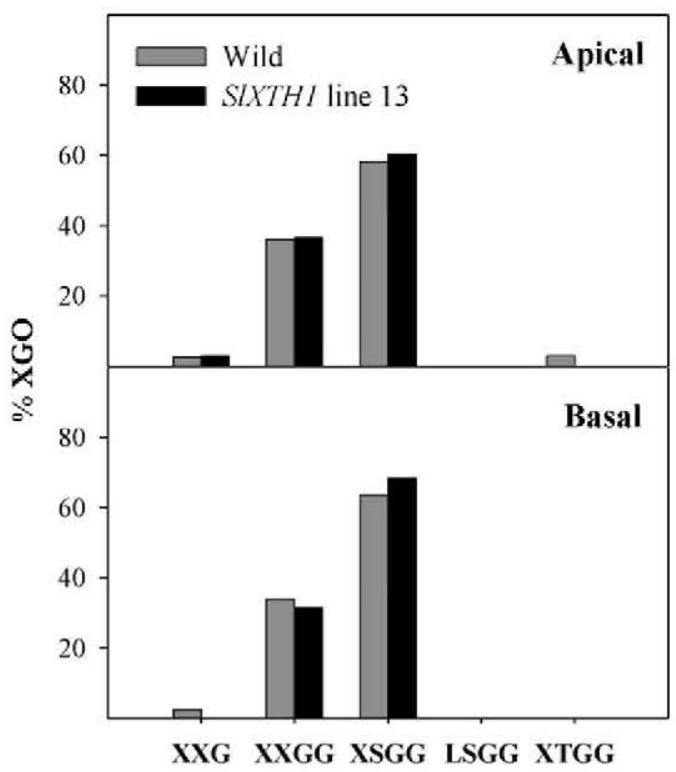

Figure 9. Relative composition of xyloglucan oligosaccharides (XGOs) from apical and basal hypocotyls segments of wild type and over-expression transgenic tomato line by MALDITOF-MS. Values shown are means of 2 independent experiments using 40 hypocotyls from each determination. 
as hydrolase (XEH) and hydrolyzing one xyloglucan molecule (Nistitani, 1997; Rose et al., 2002; Fry, 2004). This dual biochemical function is suggested to be the reason that XTH has roles in different physiological processes, as has been reported by Rose et al. (2002). It has been also suggested that XTHs, via their effect on xyloglucan metabolism, are involved in the cell expansion process (Takeda et al., 2002, Kaku et al., 2004). In the present study, we focused on cell elongation and cell wall extensibility, as in spite of the correlations between XET activity and gene expression with respect to growth, there are still many unanswered questions regarding the way XETs action could be modifying cell wall loosening during cell growth. We designed transgenic plants with higher and lower expression of SIXTH1 to produce plants with increased/decreased total XET activity, and to determine whether the modification in xyloglucan transglucosylase activity would affect hypocotyl growth, cell wall extensibility, and cell wall structure. SIXTH1 belongs to the group I/II XTHs that are thought to exclusively mediate XET activity (Baumann et al., 2007).

Our results on expression of SIXTH1 in the wild type tomato hypocotyl (Figure 3) showed a clear relationship between hypocotyl growth capacity and SIXTH1 expression, as the relative expression was much higher in the apical hypocotyl segment, where the elongation and growth is higher, than in the basal hypocotyls segment, where the growth is much lower. These results are in agreement with previously published data, since SIXTH1 has been previously associated with growth (Catalá et al., 2001). The expression of SIXTH1 in the transgenic hypocotyls showed significant over-expression of SIXTH1 (line 13) in comparison with the wild type, while co-suppression of SIXTH1 (line 33) resulted in a significant decrease of expression in the apical part. Also, the expression pattern, as noted above for the wild type, was correlated with hypocotyl growth. These results for SIXTH1 expression are in agreement with the results on specific XET activity (Figure 4). We found a significant increase in activity in the over-expression line and a significant decrease in activity in the co-suppression line compared with the wild line, with differences due mainly to the soluble XET. Thus, we suggest that SIXTH1 translates mainly for a soluble XET isoenzyme.

In spite of the differences in XET activity between the wild and the transgenic lines, we found no differences in hypocotyl growth (Figure 5). However, when we determined the hypocotyl cell wall extensibility (Figure 6), we found a relationship between the amount of XET-specific activity and cell wall extensibility. The highest total extensibility was found in the apical hypocotyl segment of the over-expressing SIXTH1 line, where the XET-specific activity and the hypocotyl growth were also highest as compared with the wild type line. On the other hand, in the co-suppression SIXTH1 line, total extensibility values were lower than in the wild type line.

To further investigate how increased or decreased levels of XET-specific activities in the transgenic lines could have altered the cell wall structure, we determined the linkages between cell wall polysaccharides by FTIR (Figs. 7 and 8). These results showed that hypocotyls over-expressing SIXTH1 and having a higher XETspecific activity (line 13) were grouped away from the wild line. The differences were found mainly in the pectins and in the cellulosexyloglucan regions, indicating that the linkages between pectins and also between cellulose and xyloglucans might be different. These results suggest that the action of the increased XET activity in transgenic line 13 could be responsible for the cell wall structural changes, and therefore, alter the cell wall extensibility in the hypocotyls. As suggested by Vissenberg et al. (2005), the action of XET on the xyloglucan-cellulose linkages could regulate cell wall extensibility and plant growth.

We also studied qualitative components of xyloglucan by mass spectrometry analysis by analyzing the xyloglucan oligosaccha- rides composition of the xyloglucan in the wild type and the over-expressing transgenic line by MALDI TOF-MS (Figure 9). However, the results showed no differences in the XGOs composition of either line, indicating that the xyloglucan structure was not affected by XET action.

However, tomato plants with altered levels of XET activity, XTH over-expressing or co-suppressed plants, did not show differences in hypocotyl growth. These results provide evidence that XTHs from group I are mainly involved in the restructuring of the cell wall during growth and development, as suggested by Van Sandt et al., 2006, but they are not the limiting factor for plant growth, although XTHs has been considered as primarily cell wall loosening agents.

\section{Acknowledgments}

This work was funded by MCYT BFU2005-08770-C02 and GVA CTBPRB/2002/377 and GVA PROMETEO/2009/075. We wish to thank Mr. D.A. Lindsay for correcting the English version of the manuscript.

\section{References}

Antosiewicz DM, Purugganan MM, Polisensky DH, y Braam J. Cellular localizat ion of Arabidopsis xyloglucan endotransglycosylase-related proteins during development and after wind stimulation. Plant Physiol 1997:115:1319-28.

Barrachina $C$, Lorences EP. Xyloglucan endotransglycosylase activity in pine hypocotyls: intracellular localization and relationship with endogenous growth. Physiol Plant 1998;102:55-60.

Baumann MJ, Eklof IM, MichelG, Kallas AM, Teeri TT, Czizek M, Brumer H. Structural Evidence for the Evolution of Xyloglucanase Activity from Xyloglucan EndoTransglycosylases: Biological Implications for Cell Wall Metabolism. Plant Cell 2007; 19:1947-63.

Bourquin V, Nishikubo N, Abe $\mathrm{H}$, Brumer $\mathrm{H}$, Denman S, Eklund $\mathrm{M}$, Christiernin $\mathrm{M}$, Teeri TT, Sundberg B, Mellerowicz EJ. Xyloglucan endotransglycosylases have a function during the formation of secondary cell walls of vascular tissues. Plant Cell 2002;4:3073-88.

Bradford MM. A rapid and sensitive method for quantification of microgram quantities of protein utilizing the principle of protein-dye binding. Anal Biochem $1976: 72: 248-52$.

Carpita NC, Gibeaut DM. Structural models of primary cell walls in flowering plants: consistency of molecular structure with the physical properties of the walls during growth. Plant ] 1993;3:1-30.

Catalá $C$, Rose JK, Bennett $A B$. Auxin regulation and spatial localization of an endo1-4-?-D-glucanase and a xyloglucan endotransglycosylase in expanding tomato hypocotyls. Plant J 1997;12:417-26.

Catalá C, Rose JK, York WS, Albersheim P, Darvill AG, Bennett AB. Characterization of a tomato xyloglucan endotransglycosylase gene that is down-regulated by auxin in etiolated hypocotyls. Plant Physiol 2001;127:1180-92.

Cosgrove DJ. Enzymes and other agents that enhance cell wall extensibility. Annu Rev Plant Physiol Plant Mol Biol 1999;50:391-417.

Fry SC, Smith RC, Renwick KF, Martin DJ, Hodge SK, Matthews KJ. Xyloglucan endotransglycosylase, a new wall-loosening enzyme activity from plants. Biochem J 1992;282:821-8.

Fry SC. Tansley Review: Primary cell wall metabolism: tracking the careers of wall polymers in living plant cells. New Phytol 2004;161:641-75.

Hayashi T. Xyloglucans in the primary cell wall. Annu Rev Plant Physiol 1989;40:139-68.

Herbers K, Lorences EP, Barrachina C, Sonnewald U. Functional characterisation of Nicotiana tabacum xyloglucan endotransglycosylase (NtXET-1): generation of transgenic tobacco plants and changes in cell wall xyloglucan. Planta 2001:212:279-87.

Herbers K, Prat S, Willmitzer L. Functional analysis of a leucine aminopeptidase from Solamum tuberosum L. Planta 1994;194:230-40.

Ji SH, Lu YC, Feng JX, Wei G, Li J, Shi YH, Fu O Liu D, Luo JC, Zhu YX. Isolation and analysis of genes preferentially expressed during early cotton fibre development by subtractive PCR and cDNA arrays. Nucleic Acids Research 2003;31:2534-43.

Kaku T, Tabuchi A, Wakabayashi K, Hoson T. Xyloglucan oligosaccharides cause cell wall loosening by enhancing xyloglucan endotransglucosylase/hydrolase activity in azuki bean epicotyls. Plant Cell Physiol 2004;45:77-82.

Kemsley EK. Discriminant analysis of spectroscopic data. (John Wiley and Sons). Principal Components Analysis of the FTIR spectra was performed using the WINDAS. Software package, 1998.

Lorences EP, Fry SC. Xyloglucan oligosaccharides with at least two R-D-xylose residues act as an acceptor for xyloglucan endotransglycosylase and promote the depolymerization of xyloglucan. Physiol Plant 1993;88:105-12.

Ling HQ, Kriseleit D, Ganal MW. Effect of ticarcillin/potassium clavulanate on callus growth and shoot regeneration in Agrobacterium-mediated transformation of tomato (Lycopersicon esculentum Mill.). Plant Cell Rep 1998;17:843-7. 
Matsui A Yokoyama R, Seki M, Ito T, Shinozaki K, Takahashi T, Komeda Y, Nishitani K AtXTH27 plays an essential role in cell wall modification during the development of tracheary elements. Plant I 2005;42:525-34.

McQueen-Mason SI, Fry SC, Durachko DM, Cosgrove DJ. The relationship between xyloglucan endotransglycosylase and in-vitro cell wall extension in cucumber hypocotyls. Planta 1993;190:327-31.

Miedes E, Lorences EP. Xyloglucan endotransglucosylase/hydrolases (XTHs) during tomato fruit growth and ripening. J Plant Physiol 2009;166:489-98.

Nishitani K, Tominaga R. Endo-Xyloglucan transferase, a novel class of glycosyltransferase that catalyzes transfer of a segment of xyloglucan molecule to another xyloglucan molecule. J Biol Chem 1992;267:21058-64.

Pritchard J, Hetherington PR, Fry SC, Tomos A. Xyloglucan endotransglycosylase activity, microfibril orientation and the profiles of cell wall properties along growing regions of maize roots. J Exp Bot 1993;44:1281-9.

Robin S, Lecomte M, Höfte $H$, Mouille $G$. A procedure for the clustering of cell wall mutants in the model plant Arabidopsis based on Fourier-transform infrared (FT-IR) spectroscopy. J Appl Stat 2003;30:669-81.

Rose JKC, Bennet AB. Cooperative disassembly on the cellulose-xyloglucan network of plant cell walls: parallels between cell expansion and fruit ripening. Trends Plant Sci 1999;4:176-83.

Rose JK, Braam J, Fry SC, Nishitani K. The XTH family of enzymes involved in xyloglucan endotransglucosylation and endohydrolysis: current pers pectives and a new unifying nomenclature. Plant Cell Physiol 2002;43:1421-35.

Saladie M, Rose JK, Cosgrove DJ, Catala C. Characterization of a new xyloglucan endotransglucosylase/hydrolase (XTH) from ripening tomato fruit and implications for the diverse modes of enzymic action. Plant J 2006;47:282-95.

Sene C, McCann MC, Wilson RH, Grinter R. Fourier-transform raman and fourier transform infrared spectroscopy (an investigation of five higher plant cell walls and their components). Plant Physiol 1994;106:1623-31.

Soga K, Wakabayashi K, Hoson T, Kamisaka S. Gravitational force regulates elongation growth of arabidopsis hypocotyls by modifying xyloglucan metabolism. Adv Space Res 2001;27:1011-6.
Taiz L Plant cell expansion: regulation of cell wall mechanical properties. Annu Rev Plant Physiol 1984;35:585-657.

Takeda T, Furuta Y, Awano T, Mizuno K, Mitsuishi Y, Hayashi T. Suppression and acceleration of cell elongation by integration of xyloglucans in pea stem segments. Proc Natl Acad Sci U S A 2002;99:9055-60.

Thompson JE, Fry SC. Restructuring of wall-bound xyloglucan by transglycosylation in living plant cells. Plant J 2001;26:23-34

Van Sandt VS, Guisez Y, Verbelen IP, Vissenberg K. Analysis of a xyloglucan endotransglycosylase/hydrolase (XTH) from the lycopodiophyte Selaginella kraussiana suggests that XTH sequence characteristics and function are highly conserved during the evolution of vascular plants. J Exp Bot 2006:57: 2909-22.

Vissenberg K, Martinez-Vilchez IM, Verbelen JP, Miller JG, Fry SC. In vivo colocalization of xyloglucan endotransglycosylase activity and its donor substrate in the elongation zone of Arabidopsis roots. Plant Cell 2000;12:1229-37.

Vissenberg K, Fry SC, Verbelen JP. Root hair initiation is coupled to a highly localized increase of xyloglucan endotransglycosylase action in Arabidopsis roots. Plant Physiol 2001:127:1125-35.

Vissenberg K, Fry SC, Pauly M, Hofte H, Verbelen JP. XTH acts at the microfibril-matrix interface during cell elongation. J Exp Bot 2005;56:673-83.

Wu Y, Spollen WG, Sharp RE, Hetherington PR, Fry SC. Root growth maintenance at low water potentials (increased activity of xyloglucan endotransglycosylase and its possible regulation by abscisic acid). Plant Physiol 1994;106: $607-15$

Wu, Sharp RE, Durachko DM, Cosorove D], Growth maintenance of the maize primary root at low water potentials involves increases in cell-wall extension properties, expansin activity, and wall susceptibility to expansins. Plant Physiol 1996; 111:765-72.

Yokoyama R, Nishitani KA Comprehensive expression analysis of all members of a gene family encoding cell-wall enzymes allowed us to predict cis-regulatory regions involved incell-wall construction in specific organs of Arabidopsis. Plant Cell Physiol 2001:42:1025-33. 